\title{
Detection and classification characteristics of arrays of carbon black/organic polymer composite chemiresistive vapor detectors for the nerve agent simulants Dimethylmethylphosphonate and
}

\section{Alan R. Hopkins, Nathan Saul Lewis}

Alan R. Hopkins, Nathan Saul Lewis, "Detection and classification characteristics of arrays of carbon black/organic polymer composite chemiresistive vapor detectors for the nerve agent simulants Dimethylmethylphosphonate and Diisopropy," Proc. SPIE 4722, Chemical and Biological Sensing III, (24 June 2002); doi: 10.1117/12.472252 


\title{
Detection and Classification Characteristics of Arrays of Carbon Black/Organic Polymer Composite Chemiresistive Vapor Detectors for the Nerve Agent Simulants Dimethylmethylphosphonate and Diisopropylmethylphosponate
}

\author{
Alan R. Hopkins and Nathan S. Lewis ${ }^{\mathrm{a}}$, \\ ${ }^{a}$ Division of Chemistry and Chemical Engineering, Noyes Laboratory, 127-72 \\ California Institute of Technology, Pasadena, CA 91125
}

\begin{abstract}
Arrays of conducting polymer composite vapor detectors have been evaluated for performance in the presence of the nerve agent simulants dimethylmethylphosphonate (DMMP) and diisopropylmethylphosponate (DIMP). Limits of detection for DMMP on unoptimized carbon black-organic polymer composite vapor detectors in laboratory air were estimated to be $0.047-0.24 \mathrm{mg} \mathrm{m}-3$. These values are lower than the EC50 value (where EC50 is the airborne concentration sufficient to induce severe effects in 50\% of those exposed for 30 minutes) for the nerve agents sarin (methylphosphonofluoridic acid, (1methylethyl) ester) and soman (methylphosphonofluoridic acid, 1,2,2-trimethylpropyl ester), which have been established as $\approx 0.8 \mathrm{mg} \mathrm{m}-3$. Arrays of these vapor detectors were easily able to resolve signatures due to exposures to DMMP from those due to DIMP or due to a variety of other test analytes (including water, methanol, benzene, toluene, diesel fuel, lighter fluid, vinegar and tetrahydrofuran) in a laboratory air background. In addition, DMMP at $27 \mathrm{mg} \mathrm{m}-3$ could be detected and differentiated from the signatures of the other test analytes in the presence of backgrounds of potential interferents in the background ambient, including water, methanol, benzene, toluene, diesel fuel, lighter fluid, vinegar and tetrahydrofuran, even when these interferents were present in much higher concentrations than that of the DMMP or DIMP being detected.Thin films of carbon black-organic polymer composites have been deposited across two metallic leads, with sorption of vapors producing swelling-induced resistance changes of the detector films. To identify and classify vapors, arrays of such vapor sensing elements have been constructed in which each element of the array contains a different polymer as the insulating phase and a common conductor, carbon black, as the conducting phase. The differing gas-solid partition coefficients for the various polymers of the detector array produce a pattern of differential resistance changes that is used to classify vapors and vapor mixtures. The performance of this detector array system towards 2,4-dinitrotoluene, the predominant signature in the vapor phase above land mines, in the presence high concentrations of water or of acetone (as a selected volatile organic carbon vapor), has been evaluated.
\end{abstract}

Keywords: Nerve agent simulants, vapor detectors, chemiresistors.

\section{INTRODUCTION}

Arrays of chemically sensitive resistors fabricated from composites of carbon black with insulating organic polymers have received significant attention recently for use in detecting, quantifying, and discriminating amongst various organic vapors. ${ }^{1-4}$ One of the potential advantages of this approach to vapor sensing ${ }^{5-20}$ is that it affords the possibility of fabricating very small size, low power, and lightweight detector arrays that have hundreds of compositionally different detector pixels. For example, hundreds of such detectors have been deposited and read out in our laboratory on a Si chip having an area of $0.04 \mathrm{~cm}^{2} ; 21-22$ thus one could envision developing wearable badge-type detectors for personal environmental monitoring devices based on this technology.

In this work, we have evaluated the detection and discrimination capabilities of an array of carbon black-organic polymer composite chemiresistors towards the nerve agent simulants dimethylmethylphosphonate (DMMP) $)^{23-24}$ and diisopropylmethylphosphonate (DIMP) (Figure 1). Table 1 indicates some chemical warfare agents and concentrations of interest. ${ }^{25}$ DMMP is often regarded as a simulant for sarin (methylphosphonofluoridic acid, (1-methylethyl) ester) and DIMP as a simulant for soman (methylphosphonofluoridic acid, 1,2,2-trimethylpropyl ester), so DMMP and DIMP have been the 
focus of our initial efforts in this area. In this work, we report the detection limits of these detector arrays, under controlled laboratory conditions, for DMMP or DIMP in background air, as well as for DMMP or DIMP in the presence of a variety of different background ambients including water vapor, diesel fuel, organic solvent vapors, and other selected possible interferents. Additionally, we have evaluated the ability of arrays of these detectors to discriminate between DMMP, DIMP, and a collection of other selected organic vapors under the measured test conditionsPrior work in our laboratory has utilized broadly responsive detector arrays based on carbon black composites ${ }^{1-3}$ for various vapor detection tasks. In this approach, films consisting of carbon black dispersed into different insulating organic polymers form the individual detector elements. The carbon black endows electrical conductivity to the films, whereas the different organic polymers provide the source of chemical diversity between elements in the detector array. Swelling of polymers ${ }^{4-11}$ upon exposure to a vapor increases the resistance of the film, thereby providing a remarkably simple means for monitoring the presence of a vapor. ${ }^{1-3}$ Because different polymer compositions are present in each detector element, an array of elements responds to a wide variety of vapors (or binary mixtures of vapors) in a distinctive, identifiable fashion. The electrical resistance signals that comprise the output from the array can be readily integrated into software or hardware-based decision systems, allowing for an integration of sensing and analysis functions into a compact, low-power, device.<smiles>COP(C)(=O)OC</smiles>

(a)<smiles>CC(C)OP(C)(=O)OC(C)C</smiles>

(b)

Figure 1. Chemical Structures of the nerve gas dimethyl methyl phosphonate (a), diisoproplyl methyl phosphonate (b).

Table 1. Human Exposure Guidelines for Selected Chemical Warfare Agents, $\mathrm{mg} \mathrm{m}^{-3}$

$\underline{\text { Agent }}$

sarin (methylphosphonofluoridic acid, (1-methylethyl) ester)

soman (methylphosphonofluoridic acid, 1,2,2-trimethylpropyl ester)

tabun (dimethylphosphoramidocyanidic acid, ethyl ester)

VX (methylphosphonothioic acid, S-[2-[bis

(1-methylethyl)amino]ethyl]- $O$-ethyl ester)

sulfur mustard

$\left(1,1^{\prime}\right.$, thiobis[2,chloroethane])

${ }^{a}$ AEL: The maximum airborne exposure concentration for an 8-hr workday.

${ }^{b} \mathrm{EC}_{50}$ : The airborne concentration sufficient to induce severe effects in $50 \%$ of those exposed for 30 minutes.

${ }^{c} \mathrm{ED}_{50}$ : The amount of liquid agent on the skin sufficient to produce severe effects in $50 \%$ of the exposed population.
$\mathrm{AEL}^{\mathrm{a}}$
$\underline{\mathrm{EC}}_{\underline{5}}^{\mathrm{b}}$
$\underline{\mathrm{ED}}_{50}{ }^{\mathrm{c}}$
0.0001
$<0.8$
1000
0.00003
$<0.8$
200
0.0001
$<1.7$
$<880$
0.00001
$<0.3$
$<2.5$
3.33
600

\section{EXPERIMENTAL}

The carbon black used in the composites was Black Pearls 2000 (BP2000), a furnace black material that was generously donated by Cabot Co. (Billerica, MA). The polymer used in the composites (Table 2) was (detector \#, polymer, manufacturer): 1, poly(ethylene oxide), Polysciences; 2, poly(55\% ethylene-co-45 \%vinyl acetate), Polysciences; 3, poly(72 \%butadiene-co-28\% styrene), Scientific Polymer Products; 4, poly(vinyl carbazole), Polysciences, 5, Poly(vinyl acetate), Scientific Polymer Products; 6, poly(caprolactone), Polysciences; 7, polysulfone, Polysciences; 8 poly(vinyl pyrrolidone), 
Scientific Polymer Products; 9, poly(4-vinyl phenol), Polysciences; 10, poly(methyloctadecyl siloxane), Polysciences. To form contacts on the substrate, two parallel bands of gold, 50-100 nm thick and separated by $1 \mathrm{~mm}$, were deposited onto conventional $7.5 \mathrm{~cm}$ X $2.5 \mathrm{~cm}$ glass slides (Corning Inc.). The slides were then cut into strips to produce $0.7 \mathrm{~cm} \times 2.5 \mathrm{~cm}$ pieces of glass. The detector films were made from a solution of the polymer into which carbon black had been suspended. $160 \mathrm{mg}$ of one of the insulating polymers was dissolved in $20 \mathrm{~mL}$ of solvent and $40 \mathrm{mg}$ of carbon black was then suspended in this solution, to produce a composition of $80 \%$ polymer and $20 \%$ carbon black by weight of solids. The solvent was either toluene or tetrahydrofuran, depending on the solubility of the polymer. The solutions were sonicated for 5 min to suspend the carbon black. A single solution that contained the polymer and the carbon black was used to prepare all the detectors of a given composition that were used in this work. An aliquot of the suspension was spin coated, at $1500 \mathrm{rpm}$, onto a glass substrate using a Headway (Garland, TX) spin coater, and the resulting film was allowed to dry in air for 24 hours. Multiple coatings of the suspension were applied to each substrate to yield detectors having resistance values of approximately a few hundred $\mathrm{k} \Omega$.

\title{
Table 2. Polymers Used in the Carbon Black-Polymer Composite Detector Array
}

\begin{tabular}{c} 
detector no. \\
\hline 1 \\
2 \\
3 \\
4 \\
5 \\
6 \\
7 \\
8 \\
9 \\
10
\end{tabular}

\author{
polymer \\ poly(ethylene oxide), PEO \\ poly(ethylene-co-vinyl acetate), $45 \%$ vinyl acetate, PEVA \\ poly(butadiene-co-styrene), $72 \%$ butadiene, PBS \\ poly(vinyl carbazole), PVC \\ poly(vinyl acetate), PVA \\ poly(caprolactone), PCL \\ polysulfone \\ poly(vinyl pyrrolidone), PVP \\ poly(4-vinyl phenol), PVPH \\ poly(methyloctadecyl siloxane), PMODS
}

The solvents used in this study were tetrahydrofuran (THF), benzene, methanol, toluene, dimethylmethylphosphonate (DMMP) and diisopropylmethylphosponate (DIMP). These solvents were purchased from Aldrich and were used without further purification. The vinegar (Lucky Brand), lighter fluid (butane and other low molecular weight hydrocarbons) and diesel fuel (Local 76) were purchased from commercial consumer sources.

The apparatus used to generate known concentrations of organic vapors and to acquire resistance vs. time data from the detectors has been described previously. ${ }^{1,26}$ Briefly, solvents were saturated with a background of laboratory air and the resultant flowing gas stream was mixed with an independently controlled flow of air to produce the desired diluted flow of analyte vapor. The gas flow rates were controlled with calibrated mass flow controllers, and analyte saturation of the flow stream that passed through the solvent bubblers was verified by measurement of the rate of mass loss of the solvent in the bubbler. ${ }^{27}$ Solenoids and one-way valves were used to mix the gases and to direct them to a Teflon chamber that contained the conducting polymer composite detectors. ${ }^{28}$ The performance of the entire vapor generation system was validated using a flame ionization detector to determine the concentration of a series of standard mixtures of toluene in air that were delivered to the detector chamber. These values were within $90 \%$ of the values expected based on the vapor pressure of the analyte and the dilution ratio expected from the settings of the mass flow controllers. The flame ionization detector was calibrated using standard gas calibration mixtures obtained from commercial suppliers.

The carrier gas for all experiments was oil-free air, obtained from the general compressed laboratory source which contained $1.10 \pm 0.15$ ppth (parts per thousand) of water vapor. The air was filtered to remove particulates, but deliberately was not dehumidified nor otherwise purified. When water was used as the background analyte, the carrier gas was ultra-zero air. Fluctuations in laboratory temperature, $21.5 \pm 1.5^{\circ} \mathrm{C}$, could cause an approximate $10 \%$ error in setting and controlling the vapor concentrations between nominally identical exposures over the course of the data collection analyzed in this work. No temperature control of the apparatus or of the carbon black/polymer composite detectors was performed. The flow rate of the vapor stream entering the exposure chamber ( 1 liter in total volume) was maintained at $15 \mathrm{~L} \mathrm{~min}^{-1}$.

The 20-detector array studied in this work had 2 nominally identical copies of each of 10 different polymer composites (Table 2). To initiate an experiment, the detectors were placed into the flow chamber and a background flow of laboratory air was introduced until the resistance of the detectors stabilized. Each exposure consisted of a three-step process that began with $180 \mathrm{~s}$ of air flow to achieve a smooth baseline resistance. After this period, the detectors were exposed for $300 \mathrm{~s}$ to analyte vapor at a controlled concentration in flowing air. The analyte exposure was then followed by $420 \mathrm{~s}$ flow of clean air 
to restore the baseline resistance values. Within each experiment, every exposure was assigned a randomly-generated index number using the Microsoft Excel random number generator. The exposures were then presented to the detector array in ascending order of the assigned index values.

To obtain signatures for the different analytes of interest, each analyte was exposed to the vapor detector array in random order at a fixed value of $\mathrm{P} / \mathrm{P}^{\circ}$, where $\mathrm{P}^{\circ}$ is the vapor pressure of the analyte at room temperature and $\mathrm{P}$ is the partial pressure of the analyte. In this study, the $180 \mathrm{~s}$ baseline period was followed by $300 \mathrm{~s}$ of exposure to the test analyte at $\mathrm{P} / \mathrm{P}^{\circ}=0.010$. Representative resistance versus time response signatures of selected detectors, and a summary of the output data for different carbon black/polymer detectors exposed to DMMP in background air, are presented in Figure 2 and Table 3 , respectively.
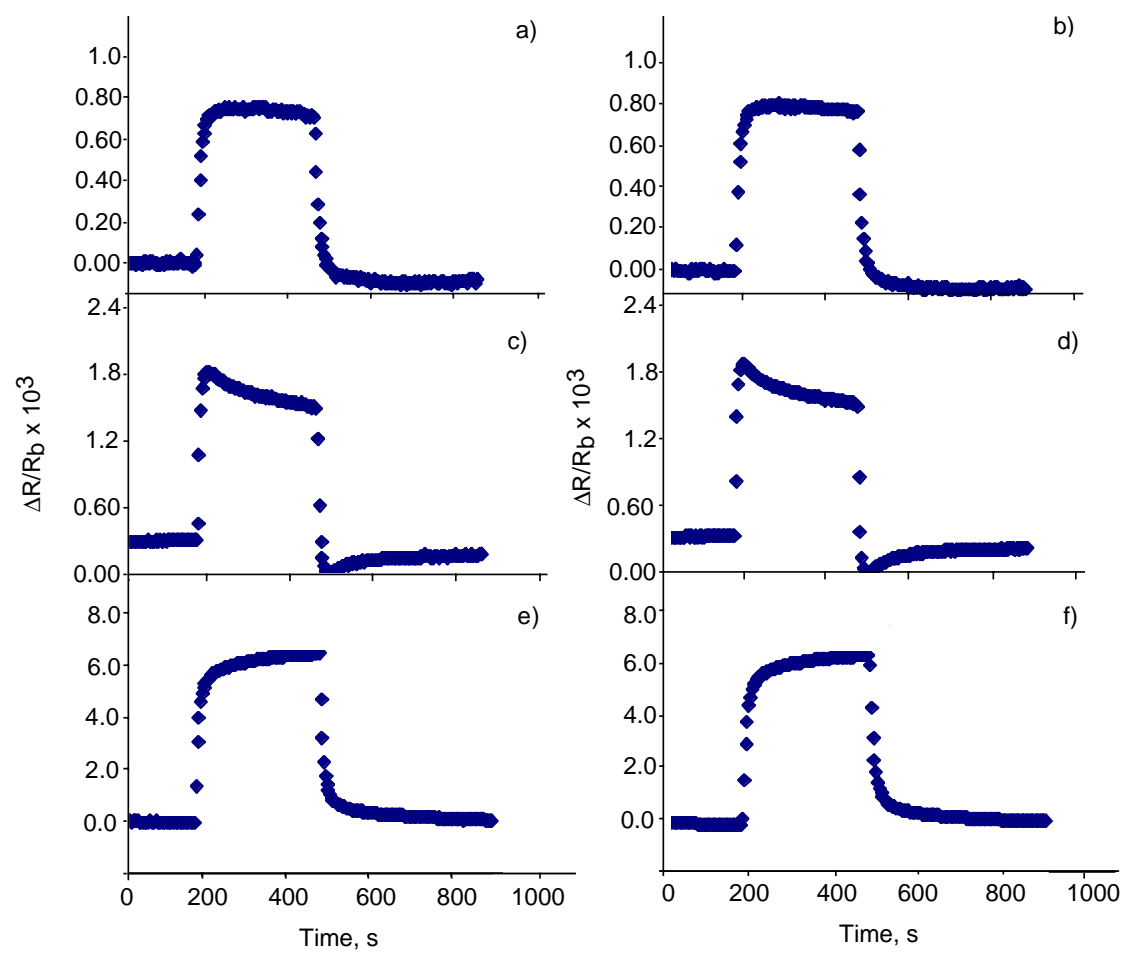

Figure 2. Response vs. time of detectors in Table 2 to DMMP.

Table 3. $\Delta R / R_{b}$ Responses of Carbon Black/Polymer Detectors to DMMP in Background Air ${ }^{a}$

\begin{tabular}{ll}
$\underline{\text { Host material }}$ & $\underline{0.0017}$ \\
\hline PEO & $(13.2 \pm 6.7) \times 10^{-5}$ \\
PEO & $(12.6 \pm 3.1) \times 10^{-5}$ \\
PEVA & $(28.7 \pm 9.8) \times 10^{-5}$ \\
PEVA & $(24.1 \pm 7.8) \times 10^{-5}$ \\
PCL & $(8.77 \pm 1.3) \times 10^{-4}$ \\
PCL & $(8.81 \pm 1.0) \times 10^{-4}$
\end{tabular}
Activity, $\mathrm{P} / \mathrm{P}^{\mathrm{o}}$ of DMMP

$\underline{0.0054}$
$(4.35 \pm 5.6) \times 10^{-4}$
$(4.56 \pm 1.2) \times 10^{-4}$
$(9.44 \pm 1.7) \times 10^{-4}$
$(9.38 \pm 2.0) \times 10^{-4}$
$(0.239 \pm 4.9) \times 10^{-4}$
$(0.242 \pm 1.8) \times 10^{-4}$

$\underline{0.013}$

$(9.20 \pm 3.4) \times 10^{-4}$

$(9.03 \pm 2.3) \times 10^{-4}$

$(0.188 \pm 4.9) \times 10^{-4}$

$(0.190 \pm 5.1) \times 10^{-4}$

$(0.675 \pm 9.8) \times 10^{-4}$ $(0.671 \pm 4.7) \times 10^{-4}$

${ }^{a}$ Values are means and standard deviations for 8 exposures of a carbon black-polymer composite detector to DMMP. Replicate entries of the host material denote the results for two detectors that were fabricated together and were exposed together to analyte. 
A separate run was performed to probe the detector response to a test analyte in the presence of various background ambients. In this run, the exposure phase consisted of two parts. The background analyte was exposed at $\mathrm{P} / \mathrm{P}^{\circ}=0.010$ for 150 $\mathrm{s}$, at which time the test analyte was introduced and exposed for an additional $150 \mathrm{~s}$. During the exposure of the second (test) analyte, the first (background) analyte was continually flowing. The foreground test analytes DMMP and DIMP were presented to the array at $\mathrm{P} / \mathrm{P}^{\circ}=0.0017,0.0054$ or 0.013 . Each unique foreground/background combination was replicated 8 times. The total experiment thus contained 192 total exposures ( 2 foreground solvents each at 3 different concentrations in the presence of a selected member of the 4 background solvents, with 8 replicates of each unique exposure combination). Although the resistance of each detector was sampled once every 3 - $5 \mathrm{~s}$ during each exposure, only the relative differential resistance change, $\Delta \mathrm{R} / \mathrm{R}_{\mathrm{b}}$ where $\Delta \mathrm{R}$ is the steady-state resistance change of the detector during exposure to the analyte and $\mathrm{R}_{\mathrm{b}}$ is the baseline resistance of the detector during the initial $180 \mathrm{~s}$ period of exposure to background air, was used in analysis of the data. The value of $\mathrm{R}_{\mathrm{b}}$ was calculated by taking the average of the last $30 \mathrm{~s}(\approx 10$ data points) of the initial $180 \mathrm{~s}$ air exposure, while $\Delta \mathrm{R}$ was calculated by averaging the last $30 \mathrm{~s}$ of data obtained during exposure of the detectors to the analyte of interest. In studies when a background analyte was introduced to the detectors prior to introduction of a foreground analyte, two separate $\Delta R / R_{b}$ values, one for the background analyte relative to clean air, and the other for the foreground analyte relative to the steady-state response of the background analyte, were calculated from the data. The value of $R_{b}$ for exposure to a foreground analyte was determined using the last $30 \mathrm{~s}$ of data recorded during exposure of the detectors to the background analyte. The $\Delta \mathrm{R} / \mathrm{R}_{\mathrm{b}}$ values were then averaged for each set of detectors over the 8 replicate exposures for each unique detector/analyte combination. The $\Delta \mathrm{R} / \mathrm{R}_{\mathrm{b}}$ values for both members of each pair of nominally identical carbon black/polymer detectors in the array were then combined to form a single average $\Delta R / R_{b}$ value for that type of polymeric detector/carbon black composite in response to the particular analyte of interest. Because only 4 detector compositions responded well to DMMP and DIMP, for consistency all PCA plots and detector performance data were evaluated using only this subset of detectors. This 8-detector array consisted of two copies of carbon black/polymer composite detectors formed using poly(ethylene oxide) (PEO), poly(ethylene-co-vinyl acetate) (PEVA), poly(butadiene-co-styrene) (PBS), and polycaprolactone (PCL), respectively.

Principal component analysis (PCA) was performed using macros written in Excel and the data were plotted using Delta Graph and Claris Works. Data were normalized over the 8-detector array for a given exposure rather than over a collection of exposures for a given detector. This normalization procedure corrects for different analyte concentrations that are a consequence of the differing vapor pressures of the test analytes. The data were not autoscaled prior to use in principal component analysis.

Data were analyzed for pairwise discrimination between analytes using the Fisher linear discriminant algorithm. ${ }^{\mathrm{xv}, \mathrm{xvi}}$ In this statistical approach, the resolution factor (rf) for any solvent pair is obtained along any vector, $\vec{w}$, from the vector projection onto $\vec{w}$ of the distance between the cluster centroids, $d_{\vec{w}}$, divided by the sum of the projected standard deviations, $\sigma_{a, \vec{w}}{ }^{2}$ and $\sigma_{b, \vec{w}}{ }^{2}$, for data arising from repeated exposures for two different analytes, $a$ and $b$. The resulting numerical resolution factor along $\vec{w}$ is defined as:

$$
r f=\frac{d w}{\sqrt{\sigma a, w_{2}+\sigma b, w_{2}}}
$$

The Fisher linear discriminant operates by searching for the vector, $\vec{w}$, such that the rf value is maximized along this optimal discriminant vector. Assuming a Gaussian distribution relative to the mean value of the data points in a given cluster, the probabilities of correctly identifying an analyte as $a$ or $b$ are approximately 72, 92 and $98 \%$ from a single presentation when analytes $a$ and $b$ are separated with resolution factors of 1.0,2.0, or 3.0, respectively. Data extracted from multiple exposures of an analyte estimate the statistical distributions of the clustered data, although extremely high rf factors might overestimate the actual array discrimination performance based on the analysis of a relatively small number of presentations (10-20) of the detectors to each analyte.

\section{RESULTS}

\section{A. Differentiation of DMMP from DIMP and Other Analytes of Interest in an Air Ambient}


Figure 3 shows data in principal component space for DMMP, DIMP, and other analytes of interest. The vapors were presented to the detectors as single component analytes at $\mathrm{P} / \mathrm{P}^{\circ}=0.010$ in a background of laboratory air. The data indicate that under such conditions, DMMP can be clearly resolved from the other analytes based on their distinct $\Delta \mathrm{R} / \mathrm{R}_{\mathrm{b}}$ response patterns on the detector array. As indicated in Table 4, resolution factors for pairwise differentiation between these pure analytes produced an average resolution factor of $\approx 90$, indicating robust resolution of DMMP from any of these other analytes under such conditions.

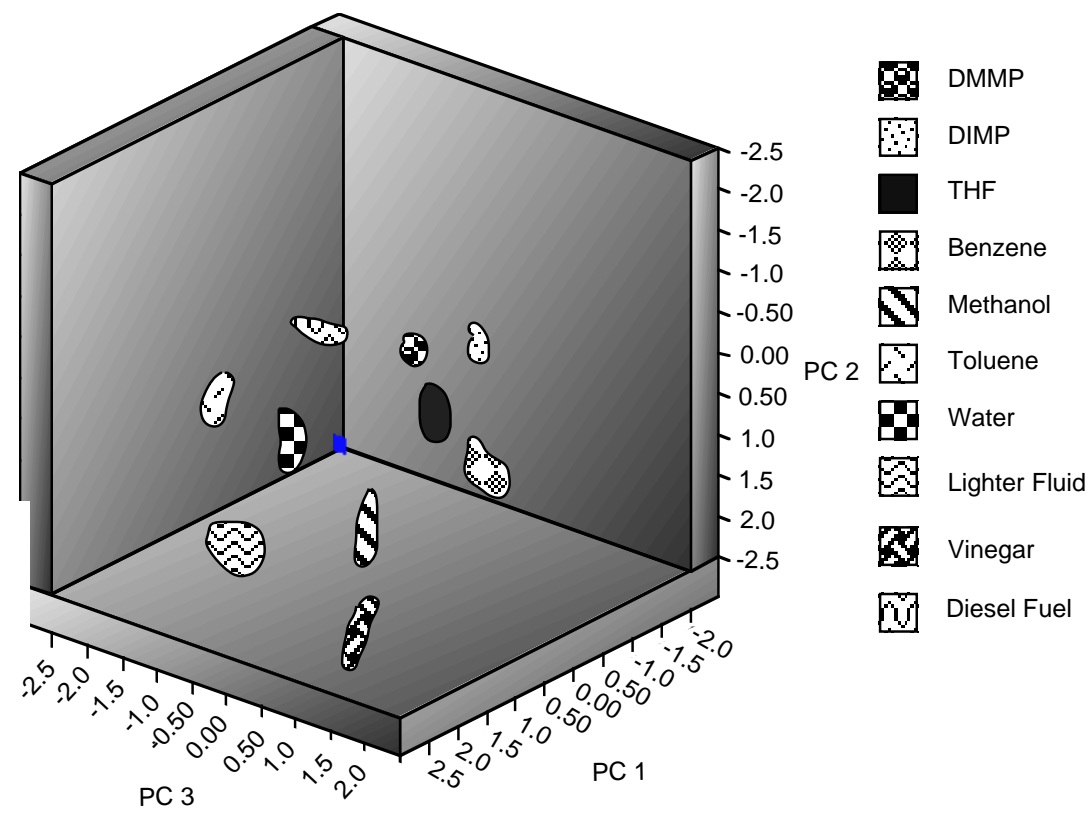

Figure 3. Separation of DMMP and DIMP from possible interferents in principal component space

\section{Table 4. Resolution Factors For an 8-Detector Array of Carbon Black Composites To Resolve Pairwise Each of the 10 Vapors, at Fixed Concentration, From Any Other Vapor in the Test Set ${ }^{\text {a }}$}

DIMP THF benzene methanol toluene water lighter fluid vinegar diesel fuel

\begin{tabular}{|c|c|c|c|c|c|c|c|}
\hline DMMP & 65 & 47 & 84 & 32 & 71 & 46 & 87 \\
\hline DIMP & 60 & 33 & 78 & 56 & 84 & 29 & 79 \\
\hline THF & & 89 & 82 & 250 & 49 & 83 & 32 \\
\hline benzene & & & 186 & 128 & 234 & 49 & 81 \\
\hline methanol & & & & 267 & 32 & 156 & 27 \\
\hline toluene & & & & & 211 & 29 & 89 \\
\hline water & & & & & & 264 & 28 \\
\hline lighter fluid & & & & & & & 87 \\
\hline vinegar & & & & & & & \\
\hline
\end{tabular}

Table 5 shows the $\Delta \mathrm{R} / \mathrm{R}_{\mathrm{b}}$ response for $\mathrm{DMMP}$ at $\mathrm{P} / \mathrm{P}^{\mathrm{o}}=0.0017,0.0054$, and 0.013 to four detectors, poly(ethylene oxide) (PEO), poly(ethylene-co-vinyl acetate) (PEVA), poly(butadiene-co-styrene) (PBS), and polycaprolactone (PCL), that responded well to the DMMP analyte. The data displayed in this table have been normalized by the value of $\mathrm{P} / \mathrm{P}^{\circ}$ and thus indicate that the pattern of response was, within experimental error, independent of the DMMP concentration. A similar dependence of the $\Delta \mathrm{R} / \mathrm{R}_{\mathrm{b}}$ detector response values vs analyte concentration has been obtained for carbon black/polymer composite chemiresistors in the presence of other vapors as well, for a comparable range of partial pressures of organic vapors in a background of laboratory air. ${ }^{31}$ 
The sensitivities, $\mathrm{S}=\left(\Delta \mathrm{R} / \mathrm{R}_{\mathrm{b}}\right) /\left(\mathrm{P} / \mathrm{P}^{\circ}\right)$, of these detectors toward DMMP and DIMP, combined with the baseline noise values for the detectors, were used to obtain detection limits for DMMP and DIMP in an air ambient on each detector type in our experimental configuration. Detection limits were taken as concentrations at which the signal/noise (calculated from the sensitivity) was $3: 1 .^{32}$ Values for these $3 \sigma$ detection values for the four most responsive detectors are summarized in Tables 6 and 7.

\begin{tabular}{|c|c|c|c|}
\hline Host material & $\underline{0.0017}$ & $\underline{0.0054}$ & $\underline{0.013}$ \\
\hline PEO & $0.0869 \pm 0.011$ & $0.0964 \pm 0.024$ & $0.0834 \pm 0.025$ \\
\hline PEVA & $0.188 \pm 0.034$ & $0.209 \pm 0.023$ & $0.170 \pm 0.029$ \\
\hline PCL & $0.577 \pm 0.053$ & $0.531 \pm 0.031$ & $0.612 \pm 0.035$ \\
\hline PBS & $0.146 \pm 0.034$ & $0.163 \pm 0.045$ & $0.133 \pm 0.021$ \\
\hline
\end{tabular}

${ }^{2}$ Values represent means and standard deviations of $\left(\Delta \mathrm{R} / \mathrm{R}_{\mathrm{b}}\right) /\left(\mathrm{P} / \mathrm{P}^{\circ}\right)$ for carbon black/polymer composite detectors in laboratory air. Data from two detectors of each polymer type were averaged together for this analysis. The analyte was exposed to the detectors 8 times at each concentration, with the concentrations randomized in the experiment.

\section{Table 6. Calculated Detection Limit of DMMP in $\mathrm{mg} \mathrm{m}^{-3}$ For Various Carbon Black-Polymer Composites}

\begin{tabular}{|c|c|c|c|c|c|}
\hline$\underline{\text { Host }}$ & \multicolumn{5}{|c|}{ Background analyte at $\mathrm{P} / \mathrm{P}^{\circ}=0.010$ in air } \\
\hline material & $\underline{\text { Air }}$ & THF & water & methanol & benzene \\
\hline PEO & 0.14 & 0.18 & 0.20 & 0.15 & 0.13 \\
\hline PEVA & 0.050 & 0.055 & 0.068 & 0.053 & 0.047 \\
\hline PCL & 0.059 & 0.051 & 0.048 & 0.062 & 0.057 \\
\hline PBS & 0.19 & 0.22 & 0.16 & 0.24 & 0.18 \\
\hline
\end{tabular}

Table 7. Calculated Detection Limit of DIMP in $\mathrm{mg} \mathrm{m}^{-3}$ For Various Carbon Black-Polymer Composites

\begin{tabular}{|c|c|c|c|c|c|}
\hline$\underline{\text { Host }}$ & \multicolumn{5}{|c|}{ Background analyte at $\mathrm{P} / \mathrm{P}^{\circ}=0.010$ in air } \\
\hline$\underline{\text { material }}$ & $\underline{\text { Air }}$ & THF & water & methanol & benzene \\
\hline PEO & 0.19 & 0.67 & 0.32 & 0.58 & 0.76 \\
\hline PEVA & 0.074 & 0.055 & 0.053 & 0.062 & 0.082 \\
\hline PCL & 0.049 & 0.039 & 0.088 & 0.051 & 0.057 \\
\hline
\end{tabular}

\section{B. Differentiation of DMMP from DIMP and Other Analytes of Interest in the Presence of Varying Background Analytes}

Figures $4 \mathrm{a}$-c show the $\Delta \mathrm{R} / \mathrm{R}_{\mathrm{b}}$ response for carbon black/polymer composite detectors that contained poly(ethylene oxide), poly(ethylene-co-vinylacetate), and polycaprolactone, respectively, to DMMP at $\mathrm{P} / \mathrm{P}^{\mathrm{o}}=0.0017,0.0054$, or 0.013 in the presence of various analytes that had been added at $\mathrm{P} / \mathrm{P}^{\mathrm{o}}=0.010$ to a laboratory air background flow. The data of Figure 4 support the data of Table 5 that the $\Delta \mathrm{R} / \mathrm{R}_{\mathrm{b}}$ response on these detectors is approximately a linear function of analyte partial pressure, and furthermore indicate that the $\Delta \mathrm{R} / \mathrm{R}_{\mathrm{b}}$ values for this range of DMMP partial pressures are, within experimental error, independent of whether the background gas was laboratory air or was laboratory air with a significant concentration of any of these other analytes. Analogous behavior was observed for DIMP. Similar behavior has also been observed previously in a general probe of the behavior of carbon black/polymer composite vapor detectors, in which $\Delta \mathrm{R} / \mathrm{R}_{\mathrm{b}}$ response patterns to various test organic vapors were observed to be essentially independent of the composition of the background ambient for a range of analyte concentrations and analyte types under laboratory test conditions. ${ }^{31}$

Figures 5 a-d shows the $\Delta \mathrm{R} / \mathrm{R}_{\mathrm{b}}$ response of four detector types to DMMP as a function of the relative humidity of the background air, when $\mathrm{P}_{\mathrm{H} 2 \mathrm{O}} / \mathrm{P}^{\mathrm{o}}{ }_{\mathrm{H} 2 \mathrm{O}}$ was varied from 0 to 0.15 at room temperature. These data are of interest for detection of DMMP and DIMP in environments in which the relative humidity is not fully controlled. The data clearly show that, within 
experimental error, the $\Delta \mathrm{R} / \mathrm{R}_{\mathrm{b}}$ response pattern for DMMP on carbon black composite detectors that contain either PEO, PEVA, PCL or PBS was independent of the relative humidity over the range of values explored in this work.

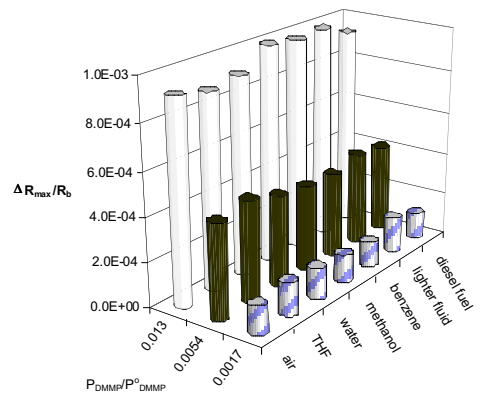

(a)

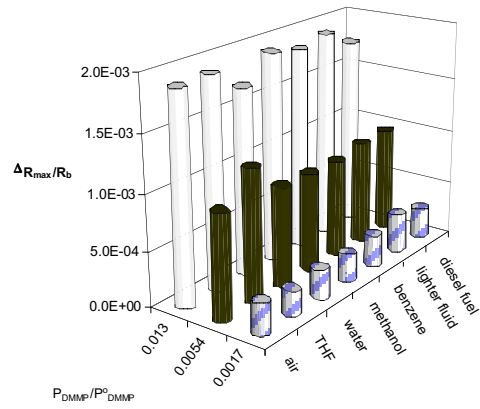

(b)

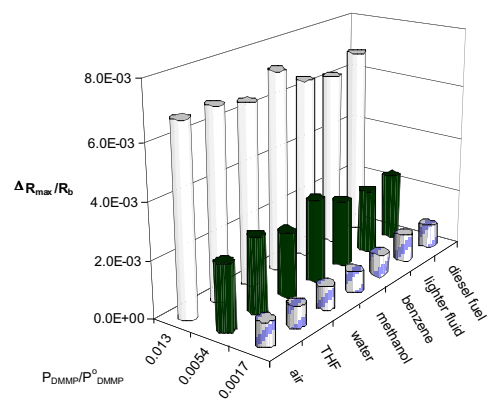

(c)

Figure 4. Responses of poly(ethylene oxide) (a), poly(ethylene-co-vinyl acetate) (b), and polycaprolactone (c) to DMMP at $\mathrm{P} / \mathrm{P}^{\circ}=0.0017,0.0054,0.013$, with various analytes added at $\mathrm{P} / \mathrm{P}^{\circ}=0.0010$ to act as interferents.

a)

b)
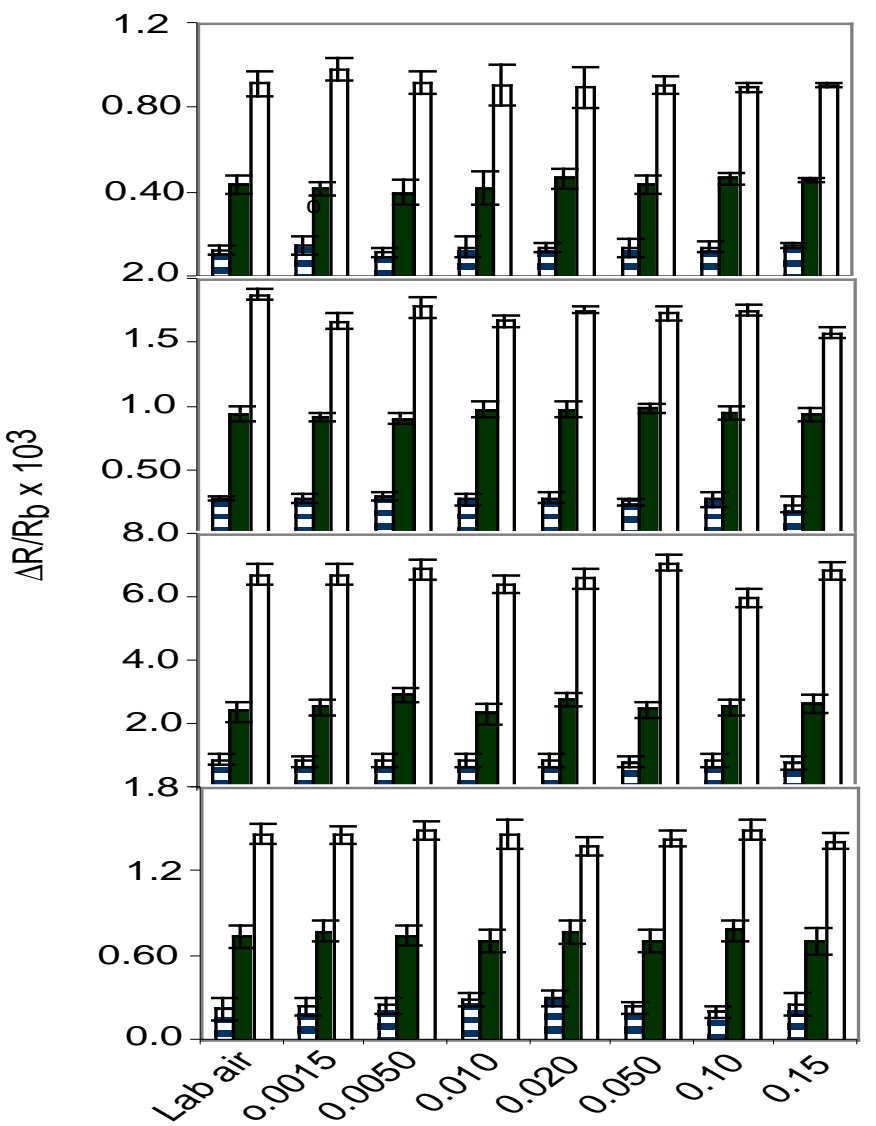

Figure 5. Responses of four different detector types to DMMP as a function of background water vapor content. $\mathrm{P}_{\mathrm{H} 2 \mathrm{O}} / \mathrm{P}_{\mathrm{H} 2 \mathrm{O}}^{\mathrm{o}}$ varied from 0 to 0.15 . 
Figures 6 and 7 summarize, in principal component space, the results of all experiments performed in this work involving exposures of DMMP and DIMP to an 8-detector array as a function of the composition of the background ambient gas in the flow stream. Consistent with Figures 3-4, the data indicate that the pattern type for DIMP and for DMMP was preserved as the concentration of analyte increased. In addition, Figures 6 and 7 demonstrate that all of the tested concentrations of DMMP and DIMP could be clearly differentiated from the patterns produced by exposure to the other pure analytes of interest. Finally, the data of Figures 6 and 7 indicate that the DMMP and DIMP patterns were essentially unchanged regardless of the composition of the background ambient gas flow evaluated in this work. Resolution factors for the 8-detector array under various conditions of interest are presented in Tables 4 and 8.

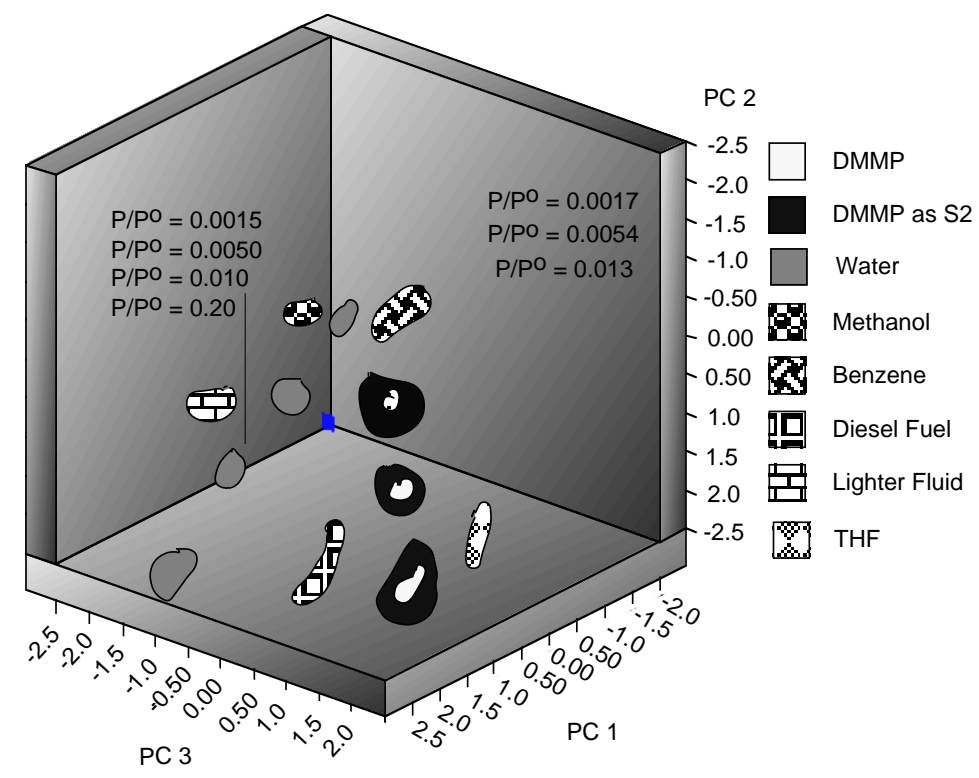

Figure 6. Separation of DMMP from potential interferents in principal component space. The darker shaded areas around the DMMP clusters represent data collected in which the DMMP was exposed along with varying background interferents.

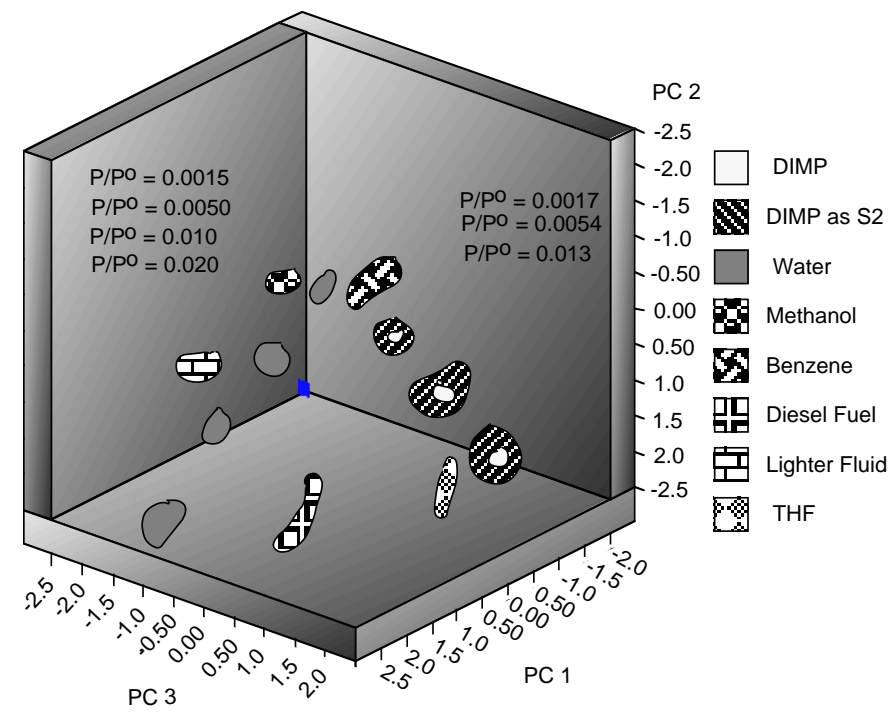

Figure 7. Separation of DIMP from potential interferents in principal component space. The darker shaded areas around the DIMP clusters represent data collected in which the DMMP was exposed along with varying background interferents.

Sensitivities and detection limits were also calculated for DMMP and DIMP in the presence of variation in the composition of the background ambient. Values for the detection limits under the various conditions studied herein are presented in 
Tables 6 and 7. As expected from the results above, the detection limits were relatively insensitive to the composition of the background analyte.

\section{Table 8. Resolution Factors For an 8-Detector Array of Carbon Black Composites To Resolve Pairwise Each of the 10 Vapors at Fixed Concentration, From Any Other Vapor in the Test Set ${ }^{a, b}$}

$\underline{\text { DIMP }}_{\mathrm{S} 2} \underline{\mathrm{THF}} \underline{\text { benzene }} \underline{\underline{\text { methanol }}} \underline{\text { toluene }}$ water lighter fluid vinegar diesel fuel

$\begin{array}{lrrrrrrrr}\text { DMMP }_{\text {S2 }} 39 & 47 & 68 & 38 & 28 & 36 & 33 & 59 & 47 \\ \text { DIMP }_{\text {S2 }} & 58 & 36 & 67 & 52 & 79 & 36 & 82 & 42 \\ \text { THF } & & 89 & 82 & 250 & 49 & 83 & 32 & 109 \\ \text { benzene } & & & 186 & 128 & 234 & 49 & 81 & 37 \\ \text { methanol } & & & & 267 & 32 & 156 & 27 & 43 \\ \text { toluene } & & & & & 211 & 29 & 89 & 25 \\ \text { water } & & & & & & 264 & 28 & 34 \\ \text { lighter fluid } & & & & & & & 87 & 28 \\ \text { vinegar } & & & & & & & & 96\end{array}$

${ }^{\mathrm{a}}$ The average and worst pairwise resolution factors are 82 and 28 , respectively.

\section{DISCUSSION}

Under our experimental conditions the detection limits for DMMP were lower than the $\mathrm{EC}_{50}$ values for sarin (Table 1). The vapor pressures of sarin and DMMP are $1.61 \times 10^{4} \mathrm{mg} \mathrm{m}^{-3}$ and $1.45 \times 10^{4} \mathrm{mg} \mathrm{m}^{-3}$ at $20^{\circ} \mathrm{C}$, respectively. ${ }^{33}$ Because the equilibrium $\Delta \mathrm{R} / \mathrm{R}_{\mathrm{b}}$ response of carbon black/polymer composite sorption detectors depends primarily on the vapor pressure of the analyte, ${ }^{34}$ the detection limits for DMMP are expected to be very similar to those for sarin.

The data clearly show that DMMP and DIMP were robustly detected and differentiated from each other at $\mathrm{P} / \mathrm{P}^{\circ}$ $=0.0017$ in the presence of various other potential "intereferents" at partial pressures of $\mathrm{P} / \mathrm{P}^{\circ}=0.010$. Because of the significant differences in vapor pressures between DMMP and DIMP and the set of interferents, the observed behavior corresponds to detection of DMMP and DIMP at the levels of 0.047-0.24 $\mathrm{mg} \mathrm{m}^{-3}$ and $0.049-0.76 \mathrm{mg} \mathrm{m}^{-3}$ respectively, in the presence of background concentrations of water, methanol, benzene, toluene, diesel fuel, lighter fluid, vinegar and THF in the range 900 to $1.6 \times 10^{3} \mathrm{mg} \mathrm{m}^{-3}$. The specific analyte concentration for $\mathrm{P} / \mathrm{P}^{0}=0.013$ of diesel fuel, vinegar, and lighter fluid have not been specified because these analytes are multi-component mixtures of analytes; nevertheless, the primary component of these analytes was present at concentrations in excess of $1.7 \times 10^{4} \mathrm{mg} \mathrm{m}^{-3}$ under our test conditions. The ability to detect rather low concentrations of DMMP in the presence of much higher concentrations of these other analytes arises primarily from the underlying thermodynamics of sorption-based detectors, as shown in detail previously, ${ }^{34}$ which favors detection of low vapor pressure analytes based on their higher partition coefficients into polymeric detector films.

It has been shown previously that the steady-state $\Delta \mathrm{R} / \mathrm{R}_{\mathrm{b}}$ response of carbon black/organic polymer composite detectors to binary mixtures of organic vapors is generally a linear combination of the steady-state $\Delta R / R_{b}$ responses for the individual components of the mixture. ${ }^{31}$ Thus, the presence of DMMP or DIMP could in principle be determined in a binary mixture of vapors exposed simultaneously to a carbon black/polymer composite detector array if there were some prior knowledge about the composition of the analyte. However, use of the steady-state $\Delta \mathrm{R} / \mathrm{R}_{\mathrm{b}}$ response patterns is best-suited for situations in which the detectors are to be utilized in change detection mode, as opposed to performing an analysis of a complex analyte mixture without any prior separation steps or temporal information on changes in vapor composition with time. In principle, an array having as many as 5-6 components can be analyzed using the equilibrium response data of a detector array containing 6-7 detectors provided that the detectors are at most partially correlated with each other. ${ }^{35}$ Additional information can be obtained, in principle, from use of the time-dependence of the detector responses, however, such data is typically not independent of the analyte background composition nor linear with changes in analyte concentration. The time response of the carbon black/polymer composite detectors is also more sensitive to variations in film thickness and carbon black content than are the steady-state $\Delta \mathrm{R} / \mathrm{R}_{\mathrm{b}}$ values used herein, so the exact performance of such a system must be evaluated for the particular task at hand and with respect to the specific implementation of the detection and sampling system used in such an application. 
In this work we have quoted limits of detection as opposed to limits of classification. ${ }^{35}$ The former are fundamental quantities that are independent of the task of concern, while the latter are intimately linked to the nature and type of the interferents, background ambients, and how well-matched a particular algorithm is to the training and test sets of data being analyzed. ${ }^{35}$ Under certain conditions and for certain tasks, limits of classification on polymer-coated surface acoustic wave detector arrays have been shown to be within a factor of 2-3 of the limits of detection, ${ }^{35}$ so the values quoted herein provide a reasonable (order-of-magnitude) estimate of the performance that might be expected in certain tasks. We also note that the detection limits presented above are only approximate performance measures for such detectors. Signal/noise ratios, and thus limits of detection, for carbon black/polymer composite chemiresistors at equilibrium with an analyte of interest have been shown to decrease as the detector area decreases, ${ }^{36}$ so our detection limits must be scaled accordingly if the detector area is varied. In addition, the sensitivity of sorption detectors decreases with increasing temperature ${ }^{35}$ so the signal $/$ noise ratio, and thus detection limits, will decrease if the detectors are heated. In comparing the performance of these chemiresistors to prior results on polymer-coated surface acoustic wave devices for detection of DMMP and other nerve agents and nerve agent simulants, ${ }^{23}$ it is important to note that we have not used any preconcentration of the sampled analyte and have only used steady-state $\Delta \mathrm{R} / \mathrm{R}_{\mathrm{b}}$ amplitudes in an attempt to characterize the fundamental performance of the detectors towards the analytes of interest. Significant tradeoffs in sensitivity, time response, power, and other system properties would of course be involved in an engineering implementation of these detectors in a fielded device for nerve agent detection. Finally, we note that the detectors used in our study were not specifically designed to possess high partition coefficients towards phosphonatecontaining analytes such as DMMP or DIMP. Work on polymeric coatings for surface acoustic wave devices has indicated that significant improvements in sensitivity are possible through use of detector films designed to have hydrogen-bonding acceptor groups that provide complimentarity to the hydrogen-bond donor groups in phosphonate-containing nerve agents. ${ }^{23,27}$ Because the sensitivity improvements in the polymer-coated surface acoustic wave devices are directly related to increases in sorption of the analytes into the polymer films, ${ }^{38,39}$ similar improvements in detection limits towards DMMP, DIMP, sarin, and soman would be expected if such polymers were incorporated into arrays of carbon black/polymer composite vapor detectors.

\section{CONCLUSIONS}

In summary, generic, untailored arrays of carbon black/polymer chemiresistive vapor detectors can detect DMMP and DIMP at levels below the $\mathrm{EC}_{50}$ limits for the nerve agents sarin and soman. DMMP can be differentiated from DIMP and from a variety of other analytes either in laboratory air or in laboratory air that contains the presence of relatively high concentrations of various types of volatile organic vapors. Concentration-normalized response patterns for DMMP and DIMP are independent of concentration and of background ambient over the range of concentrations and ambients tested in this work. Further improvements in the fundamental performance parameters of these detectors towards the analytes of interest are likely to occur through the use of specially-tailored polymers and better control over temperature and other system variables.

\section{ACKNOWLEDGMENTS}

We acknowledge the Department of Energy (DE-FG03-98NV13367), and the Army Research Office through a MURI (DAAG55-98-1-0266) and DARPA (DAAK-60-97-K9503) for support of this work. We also acknowledge helpful discussions with V. George of the Army Night Vision Laboratory regarding detection limits and background ambients of interest 


\section{REFERENCES}

1) Lonergan, M. C.; Severin, E. J.; Doleman, B. J.; Beaber, S. A.; Grubbs, R. H.; Lewis, N. S. Chem. Mater. 1996, 8, 22982312

2) Doleman, B. J.; Sanner, R. D.; Severin, E. J.; Grubbs, R. H.; Lewis, N. S. Anal. Chem. 1998, 70, $2560-2564$.

3) Swann, M. J.; Glidle, A.; Cui, L.; Barker, J. R.; Cooper, J. M. Chem Comm. 1998, 24, 2753-2754.

4) Patel, S. V.; Jenkins, M. W.; Hughes, R. C.; Yelton, W. G.; Ricco, A. J. Anal. Chem. 2000, 72, $1532-1542$.

5) Gardner, J. W.; Pearce, T. C.; Friel, S.; Bartlett, P. N.; Blair, N. Sens. Actuators B 1994, 18, $240-243$.

6) Pearce, T.; Gardner, J. Analyst 1998, 123, 2047-2055

7) Gardner, J. W.; Gardner, W.E. Insight 1997, 39, 865-869

8) Pearce, T. C.; Gardner, J.W.; Friel, S.; Bartlett, P.N.; Blair, N. Analyst 1993, 118, 371-377.

9) Baldacci, S; Matsuno, T.; Toko, K.; Stella, R.; De Rossi, D. Sens. Mater. 1998, 10, 185-200.

10) Hodgins, D.; Simmonds, D. J. Autom. Chem. 1995, 17, 179-185.

11) Hirschfelder, M.; Ulrich, D.; Hoberg, E.; Hanrieder, D. Gartenbauwissenschaft 1998, 63, 185-190.

12) Simon, J. E.; Hetzroni, A.; Bordelon, B.; Miles, G.E.; Charles, D. J. J. Food Sci. 1996, 61, 967.

13) Broda, S.; Schnitzler, W. Deut. Lebensm-Rundsch 1998, 94, 13-16.

14) Stuetz, R. M.; White, M.; Fenner, R. A. J. Water Serv. Res. Technol.-Aqua 1998, 47, 223-228.

15) Stuetz, R .M.; Engin, G.; Fenner, R.A. Water Sci. Technol. 1998, 38, 331-335.

16) Stuetz, R. M.; Fenner, R. A.; Engin, G. Water Res. 1999, 33, 453-461.

17) Stuetz, R. M. Fenner, R. A., Engin, G. Water Res. 1999, 33, 442-452.

18) Chandiok, S.; Crawley, B.; Oppenheim, B.; Chadwick, P.; Higgins, S.; Persaud, K. J. Clin. Pathol. 1997, 50, 790-791.

19) Gardner, J. W.; Craven, M.; Dow, C.; Hines, E. L. Meas. Sci. Technol. 1998, 9, 120-127.

20) Persaud, K.; Pisanelli, A.; Szyszko, S.; Reichl, M. J.; Horner, G.; Rakow, W.; Keding, H.; Wessels, H. Sens Actuators B 1994, 18, 211-220.

21) Dickson, J. A.; Freund, M. S.; Lewis, N. S.; Goodman, R. M. Solid State Sensor and Actuator Workshop, June 2-8, 2000, Hilton Head Island, SC.

22) Briglin, S. M.; Burl, M. C.; Freund, M. S.; Lewis, N. S.; Matzger, A.; Ortiz, D. N.; Tokumaru, P. Proc. SPIE 14th Annual International Symposium on Aerospace/Defence Sensing, Simulation, and Controls, 2000, Vol. 4038, in press.

23) Grate, J.W.; Rose-Pehrsson, S. L.; Venezky, D. L.; Klusty, M.; Wohltjen, H. Anal. Chem. 1993, 65, $1868-1881$.

24) Ballantine, D. S.; Rose, S. L.; Grate, J. W.; Wohltjen, H. Anal. Chem. 1986, 58, 3058-3066.

25) Chemical and Biological Terrorism Research and Development to Improve Civilian Medical Response, Institute of Medicine and National Research Council, National Academy Press, Washington, D.C. 1999.

26) Sotzing, G. A.; Briglin, S. M.; Grubbs, R. H.; Lewis, N. S. Anal. Chem. 2000, 72, 3181-3190

27) Atkins, P. W. Physical Chemistry, $5^{\text {th }}$ ed.; W. H. Freeman and Co.: New York, 1994.

28) Severin, E.J. Ph. D. Thesis, California Institute of Technology, 1999.

29) Doleman, B. J.; Lonergan M. C.; Severin, E. J.; Vaid, T. P.; Lewis N. S. Anal. Chem. 1998, 70, $4177-4190$.

30) Duda, R. O.; Hart, P. E. Pattern Classification and Scene Analysis, John Wiley \& Sons: New York, 1973.

31) Severin, E. J.; Doleman, B. J.; Lewis, N. S. Anal. Chem. 2000, 72, 658-688.

32) Skoog, D. A.; West, D. M. Fundamentals of Analytical Chemistry, $4^{\text {th }}$ ed.; Sanders College Publishing, 1982.

33) George, V.; private communication, PM-MCD, 10205 Burbeck Road, Fort Belvoir, VA 22060.

34) Doleman, B. J.; Severin, E. J.; Lewis, N. S. Proc. Natl. Acad. Sci. U.S.A., 1998, 95, 5442-5447.

35) (a) Zellers, E. T.; Han, M. Anal. Chem. 1996, 68, 2409-2418. b) Zellers, E.T.; Park, J.; Hsu, T.; Groves, W.A. Anal. Chem., 1998, 70, 4191-4201.

36) Briglin, S. M.; Freund, M. S.; Tokumaru, P.; Lewis, N. S. submitted for publication.

37) Abraham, M. H.; Whiting, G. S. J. Chromatogr. 1991, 588, 361-364.

38) Grate, J. W.; Wise, B. M.; Abraham, M. H. Anal. Chem. 1999, 71, 4544-4553.

39) Grate, J. W.; Abraham, M. H. Anal. Chem. 1996, 68, 913-917. 